\title{
An interesting new Mahonian permutation statistic
}

\author{
Mark C. Wilson* \\ Department of Computer Science, University of Auckland \\ Private Bag 92019 Auckland, New Zealand \\ mcw@cs.auckland.ac.nz
}

Submitted: Jul 21, 2010; Accepted: Oct 21, 2010; Published: Oct 29, 2010

Mathematics Subject Classification: Primary 05A05. Secondary 68W20, 68W40, 68Q25.

\begin{abstract}
The standard algorithm for generating a random permutation gives rise to an obvious permutation statistic DIS that is readily seen to be Mahonian. We give evidence showing that it is not equal to any previously published statistic. Nor does its joint distribution with the standard Eulerian statistics des and exc appear to coincide with any known Euler-Mahonian pair.

A general construction of Skandera yields an Eulerian partner eul such that (eul, DIS) is equidistributed with (des, MAJ). However eul itself appears not to be a known Eulerian statistic.

Several ideas for further research on this topic are listed.
\end{abstract}

\section{The statistic}

\section{$1.1 \quad$ Random permutations}

The standard algorithm [Knu81, 3.4.2, Algorithm P] for uniformly generating a random permutation of $[n]:=\{1, \ldots, n\}$ is as follows. Start with the identity permutation $\iota=$ $1 \ldots n$ in the symmetric group $S_{n}$. There are $n$ steps labelled $n, n-1, \ldots, 1$ (the last step can be omitted, but it makes our notation easier to include it here). At step $i$ a random position $j_{i}$ is chosen uniformly from $[i]$ and the current element in position $j_{i}$ is swapped with the element at position $i$.

Example 1. The permutation $25413 \in \mathrm{S}_{5}$ is formed by choosing $j_{5}=3, j_{4}=1, j_{3}=$ $1, j_{2}=1, j_{1}=1$. Its inverse 41532 is formed by choosing $j_{5}=2, j_{4}=3, j_{3}=2, j_{2}=$ $1, j_{1}=1$.

\footnotetext{
*Thanks to Frank Ruskey, Mark Skandera, Einar Steingrímsson and Kyle Petersen for useful discussions.
} 
In terms of multiplication in $\mathrm{S}_{n}, \pi$ is a product of "transpositions" $\prod_{i \leqslant n}\left(i j_{i}\right)$. Any of these "transpositions" may be the identity permutation. This representation as a "triangular product" gives a bijection between $\mathrm{S}_{n}$ and the set of sequences $\left(j_{1}, \ldots, j_{n}\right)$ that satisfy $1 \leqslant j_{i} \leqslant i$ for all $i$.

Knuth attributes this algorithm to R. A. Fisher and F. Yates [FY38], and a computer implementation was given by Durstenfeld [Dur64]. Recently [Wil09] the present author and others have studied the distribution of various quantities associated with the algorithm.

\subsection{The statistic}

For each $n \geqslant 1$, there is a map $\mathrm{S}_{n} \rightarrow \mathrm{S}_{n+1}$ that maps $\pi$ to the permutation $\pi^{\uparrow}$ that fixes $n+1$ and agrees on $1, \ldots, n$ with $\pi$. We let $\mathrm{S}$ be the direct limit of sets induced by these maps. If we think of each ${ }^{\uparrow}$ as an inclusion map, as is common, then $\mathrm{S}$ is simply the union of all $\mathrm{S}_{n}$. For our purposes a permutation statistic is simply a function $T: \mathrm{S} \rightarrow \mathbb{N}$.

Of course it is always possible to construct a statistic $T$ by for each $n$ making it equal to a given statistic $T_{n}$ on $\mathrm{S}_{n}$. However unless the values of $T$ cohere for different values of $n$ this is not useful. We define a statistic on $\mathrm{S}$ to be coherent if it satisfies the following property. To be coherent, the identity $T(\pi)=T\left(\pi^{\uparrow}\right)$ must hold for all $n$ and $\pi \in \mathrm{S}_{n}$.

We now define a (coherent) permutation statistic, which we denote by DIS, as follows.

Definition 2. At step $i$ of the algorithm described above, one symbol moves rightward a distance $d_{i}=i-j_{i}$ (possibly zero), and one symbol moves leftward the same distance. We define $\operatorname{DIS}(\pi)=\sum_{i} d_{i}$, the total distance moved rightward by all elements.

There is an alternative interpretation of DIS. The sequence of moves that formed $\pi$ starting from the identity will take $\pi^{-1}$ to the identity, and the moves are the same as selection sort. The algorithm then sorts $\pi^{-1}$ via selection sort. We can think of DIS as a measure of the work done by selection sort when comparisons have zero cost. This model might be useful in analysing, for example, physical rearrangement of very heavy distinct objects.

In view of the last paragraph it makes sense also to consider the statistic IDIS given by $\operatorname{IDIS}(\pi)=\operatorname{DIS}\left(\pi^{-1}\right)$.

Example 3. For our running example 25413, the value of DIS is $(1+2+3+4+5)-$ $(1+1+1+1+3)=8$, while for IDIS the value is 6 . In terms of $\pi^{-1}=41532$, the swaps used to create $\pi$ yield successively 41235, 31245, 21345, 12345.

Given a permutation for which we do not already know the $j_{i}$, we can find these easily.

Example 4. Given $\pi=25413$ as above, we can read off $j_{5}=3$ from $\pi$. Thus multiplying $\pi$ on the right by the transposition (35) leads to 23415. We have now reduced to $\pi=2341$. We now read off $j_{4}=1$ and reduce to $\pi=231$. Continuing in this way we obtain $j_{3}=1, j_{2}=1, j_{4}=1$. 
At first sight it may appear that we must search to find the position of symbol $i$ at step $i$, leading to a quadratic time algorithm for the procedure of the last example. However this is not the case, provided we compute DIS and IDIS simultaneously, and the entire computation can be done in linear time (note that computing $\pi^{-1}$ from $\pi$ is a linear time operation). Note that, for example, it is still unknown whether the number of inversions INV of a permutation can be computed in linear time.

Example 5. In the running example $\pi=25413, \pi^{-1}=41352$, we read off $j_{5}(\pi)=$ $3, j_{5}\left(\pi^{-1}\right)=2$. To multiply $\pi$ on the right by the transposition (35) we need not scan all of $\pi$, because we know the location of the symbol 5 , namely $j_{5}\left(\pi^{-1}\right)$. Thus the multiplication takes constant time. We can either multiply $\pi^{-1}$ on the left by (35) or on the right by (25). Each leads to the same answer, namely 41235, and this is the inverse of the updated $\pi$. Continuing in this way we obtain the result of the last example.

It will be helpful to know the values of DIS on some special permutations.

Example 6. We define

$$
\begin{aligned}
\pi_{0} & =n(n-1) \ldots 1 \\
\pi_{1} & =2 \ldots n 1 \\
\pi_{1}^{-1} & =n 12 \ldots(n-1)
\end{aligned}
$$

Note that $\pi_{0}$ is created by the algorithm by choosing $j_{i}=n+1-i$ provided $n+1-i<i$, whereupon all later swaps are trivial. Also $\pi_{1}$ is created by choosing $j_{i}=1$ for all $i$, while $\pi_{1}^{-1}$ is formed by choosing $j_{i}=i-1$ for $i \geqslant 2$. Thus

$$
\begin{aligned}
\operatorname{DIS}(\iota) & =0 \\
\operatorname{DIS}\left(\pi_{0}\right) & =\left\lfloor n^{2} / 4\right\rfloor= \begin{cases}\frac{n^{2}}{4} & \text { if } n \text { is even; } \\
\frac{n^{2}-1}{4} & \text { if } n \text { is odd. }\end{cases} \\
\operatorname{DIS}\left(\pi_{1}\right) & =n(n-1) / 2 \\
\operatorname{DIS}\left(\pi_{1}^{-1}\right) & =n-1
\end{aligned}
$$

The maximum value of DIS on $\mathrm{S}_{n}$ is $n(n-1) / 2$, corresponding uniquely to the $n$-cycle $\pi_{1}$. The minimum value of DIS on $S_{n}$ is 0 , corresponding uniquely to the identity $\iota$.

As a random variable, the restriction DIS ${ }_{n}$ of DIS to $S_{n}$ is the sum of DIS ${ }_{n-1}$ and a random variable $U_{n}$ that is uniform on $[0,1, \ldots, n-1]$. Thus, iterating this recurrence, we see that $\mathrm{DIS}_{n}$ has probability generating function $F_{n}(q):=\prod_{i=1}^{n} \frac{1-q^{i}}{1-q}$. This is the definition of a Mahonian statistic on $\mathrm{S}_{n}$. Note that $n(n-1) / 2-\mathrm{DIS}=\sum_{i} j_{i}$ is also Mahonian by the symmetry of the Mahonian distribution. 


\section{DIS is not trivially equal to a known statistic}

Tabulating numerical values makes it clear that DIS is not equal to any of the most wellknown Mahonian statistics. Table 1 gives the values of DIS and several other Mahonian statistics when $n=4$ (it is amusing to note that they all coincide on the element 2134 the obvious conjecture that they always coincide on $2134 \ldots n$ is in fact correct). These statistics are INV, MAJ, DEN, MAD, MAK, HAG. We recall the unified definition of these statistics given in [CSZ97]. We first require some partial statistics.

Table 1: Values of some permutation statistics for $n=4$.

$\begin{array}{cccccccc}\pi & \text { DIS } & \text { INV } & \text { MAJ } & \text { DEN } & \text { MAD } & \text { MAK } & \text { HAG } \\ 1234 & 0 & 0 & 0 & 0 & 0 & 0 & 0 \\ 1243 & 1 & 1 & 3 & 3 & 1 & 3 & 3 \\ 1324 & 1 & 1 & 2 & 2 & 1 & 2 & 2 \\ 1342 & 3 & 2 & 3 & 5 & 1 & 4 & 5 \\ 1423 & 2 & 2 & 2 & 2 & 2 & 2 & 3 \\ 1432 & 2 & 3 & 5 & 3 & 2 & 5 & 2 \\ 2134 & 1 & 1 & 1 & 1 & 1 & 1 & 1 \\ 2143 & 2 & 2 & 4 & 4 & 2 & 4 & 4 \\ 2314 & 3 & 2 & 2 & 3 & 1 & 3 & 3 \\ 2341 & 6 & 3 & 3 & 6 & 1 & 5 & 6 \\ 2413 & 4 & 3 & 2 & 3 & 2 & 3 & 4 \\ 2431 & 4 & 4 & 5 & 4 & 2 & 6 & 3 \\ 3124 & 2 & 2 & 1 & 1 & 2 & 1 & 2 \\ 3142 & 4 & 3 & 4 & 4 & 3 & 5 & 5 \\ 3214 & 2 & 3 & 3 & 2 & 2 & 3 & 1 \\ 3241 & 5 & 4 & 4 & 5 & 2 & 6 & 4 \\ 3412 & 4 & 4 & 2 & 3 & 2 & 3 & 4 \\ 3421 & 5 & 5 & 5 & 4 & 2 & 6 & 3 \\ 4123 & 3 & 3 & 1 & 1 & 3 & 1 & 3 \\ 4132 & 3 & 4 & 4 & 2 & 4 & 4 & 2 \\ 4213 & 3 & 4 & 3 & 2 & 3 & 3 & 2 \\ 4231 & 3 & 5 & 4 & 3 & 3 & 5 & 1 \\ 4312 & 5 & 5 & 3 & 4 & 3 & 3 & 5 \\ 4321 & 4 & 6 & 6 & 5 & 3 & 6 & 4\end{array}$

Definition 7. A descent is an occurrence of the event $\pi(i)>\pi(i+1)$. The index $i$ is the descent bottom and $\pi(i)$ is the corresponding descent top.

Each $\pi$ can be uniquely decomposed into descent blocks (maximal descending subwords). Denote the first and last letter of each block of length at least 2 by $c(B), o(B)$. The right embracing number of $\pi(i)$ is the number of descent blocks strictly to the right 
of the block containing $\pi(i)$ and for which $c(B)>\pi(i)>o(B)$. The sum of all right embracing numbers is denoted by $\operatorname{Res}(\pi)$.

Example 8. For $\pi_{1}$ the descent blocks are all of length 1 except for the last one, $n 1$. The right embracing number of each letter $2, \ldots, n-1$ is 1 and the right embracing number of $n$ and of 1 are each 0 . For $\pi_{1}^{-1}$ there is again a single nontrivial descent block, namely $n 1$, and all right embracing numbers are 0 . For $\pi_{0}$ there is a single descent block of length $n$ and all right embracing numbers are 0 .

Definition 9. An excedance is an occurrence of the event $\pi(i)>i$. The index $i$ is the excedance bottom and $\pi(i)$ is the corresponding excedance top. The sum of all descent/excedance tops/bottoms of $\pi$ we denote by $\operatorname{Dtop}(\pi), \operatorname{Etop}(\pi), \operatorname{Dbot}(\pi), \operatorname{Ebot} \pi$. The differences Ddif $(\pi)$ and $\operatorname{Edif}(\pi)$ are given by Ddif $=$ Dtop - Dbot, Edif $=$ Etop - Ebot.

There is a unique decomposition $\pi$ into $\pi_{E}$ and $\pi_{N}$, where $\pi_{E}$ is the subsequence formed by excedances and $\pi_{N}$ the subsequence formed by nonexcedances. For our running example $\pi=25413$, we have $\pi_{E}=25$ and $\pi_{N}=413$. For the inverse 41532 we have respectively 45 and 132 . We define $\operatorname{Ine}(\pi)=\operatorname{INV}\left(\pi_{E}\right)+\operatorname{INV}\left(\pi_{N}\right)$.

For each excedance bottom $i$ we define $\mathrm{L}(i)$ to be the number of indices $k$ such that $k<i$ and $a_{k} \leqslant i$; let $\mathrm{L}$ be the sum over all such $i$.

Example 10. Note that $\left(\pi_{1}\right)_{E}=23 \ldots n$ and $\left(\pi_{1}\right)_{N}=1$. Similarly $\left(\pi_{1}^{-1}\right)_{E}=n$ and $\left(\pi_{1}^{-1}\right)_{N}=12 \ldots(n-1)$. Also $\left(\pi_{0}\right)_{E}=n(n-1) \ldots t+1$ and $\left(\pi_{0}\right)_{N}=t \ldots 1$, where $t=\lfloor n / 2\rfloor$.

The values of the partial statistics defined above are tabulated in Table 2 .

Proposition 11 ([CSZ97]). We have

$$
\begin{aligned}
\mathrm{MAK} & =\text { Dbot }+ \text { Res } \\
\mathrm{MAD} & =\text { Ddif }+ \text { Res } \\
\mathrm{DEN} & =\text { Ebot }+ \text { Ine } \\
\mathrm{INV} & =\text { Edif }+ \text { Ine } \\
\mathrm{HAG} & =\mathrm{Edif}+\operatorname{INV}\left(\pi_{E}\right)-\operatorname{INV}\left(\pi_{N}\right)+\mathrm{L}
\end{aligned}
$$

In addition MAJ is the sum of indices corresponding to descent tops.

\subsection{Trivial bijections}

To show that statistics $T$ and $T^{\prime}$ are different, it suffices to find some $n$ and some $\pi \in \mathrm{S}_{n}$ for which $T(\pi) \neq T^{\prime}(\pi)$. However it may be the case that $T$ and $T^{\prime}$ agree on $\mathrm{S}_{n}$ for some larger values of $n$. If both $T$ and $T^{\prime}$ are coherent, this possibility cannot occur.

Note that DIS and IDIS, along with all statistics from previous literature with which we compare them here, are coherent. Thus simply computing values for small $n$, as in the 
Table 2: Values of partial statistics on special permutations $(t=\lfloor n / 2\rfloor)$

\begin{tabular}{|c|c|c|c|c|c|c|c|}
\hline perm & Ebot & Edif & Dbot & Ddif & Res & Ine & L \\
\hline$\pi_{0}$ & $t(t+1) / 2$ & $\left\lfloor n^{2} / 4\right\rfloor$ & $n(n-1) / 2$ & $n-1$ & 0 & $\left\lfloor(n-1)^{2} / 4\right\rfloor$ & 0 \\
\hline$\pi_{1}$ & $n(n-1) / 2$ & $n-1$ & 1 & $n-1$ & $n-2$ & 0 & $(n-1)(n-2) / 2$ \\
\hline$\pi_{1}^{-1}$ & 1 & $n-1$ & 1 & $n-1$ & 0 & 0 & 0 \\
\hline
\end{tabular}

previous section, is usually enough to distinguish the statistics. However we can often give a general construction of permutations for which a given pair of statistics differs greatly.

Although DIS does not equal any of the well-known statistics of the previous section, it is possible a priori that DIS has the form $S \circ g$ where $S$ is a known Mahonian statistic and $g$ is a filtered bijection of $\mathrm{S}$ (a bijection of $\mathrm{S}$ that bijectively takes $\mathrm{S}_{n}$ to $\mathrm{S}_{n}$ for each $n)$.

In this section we consider the so-called "trivial" involutions of $\mathrm{S}_{n}$ (there is a nontrivial bijection $\Phi$ of $S_{n}$ introduced in [CSZ97]; we give more details in Section 2.2.) These involutions are inversion (group-theoretic inverse), reversal (reverse the order of the letters) and complementation (subtract each letter from $n+1$ ). Then in the obvious notation $R$ and $C$ commute and $I R=C I, I C=R I$. Thus $I, R, C$ generate a group $G$ isomorphic to the dihedral group of order 8 .

For example we have

$$
\begin{aligned}
(25413)^{I} & =41532 \\
(25413)^{R} & =31452 \\
(25413)^{C} & =41253 \\
(25413)^{I C} & =25134 \\
(25413)^{I R} & =23514 \\
(25413)^{R C} & =35214 \\
(25413)^{I R C} & =41532
\end{aligned}
$$

We shall show that DIS is not trivially equivalent to any well-known statistic. In the absence of a standardized database of permutation statistics, we define "well-known" to mean "mentioned in at least one of the papers [CSZ97, BS00]". We define $\Sigma$ to be the set consisting of well-known Mahonian statistics.

In [BS00] it is shown how all known "descent-based" Mahonian statistics can be written in terms of "Mahonian $d$-functions" for some $d \leqslant 4$. Each such $d$-function simply computes the numbers of occurrences of a certain generalized permutation pattern of length at most $d$, then sums this process over a finite number of such patterns. In particular in Table 1 of the above article, all 14 Mahonian 3-functions (up to trivial bijections) are given. In [CSZ97] the images of these statistics under a bijection $\Phi$ were also considered. We consider this bijection in Section 2.2.

Theorem 12. There do not exist $S \in \Sigma$ and $g \in G$ such that DIS $=S \circ g$. 
Proof. Note that $\pi_{0}^{R}=\iota=\pi_{0}^{C}$ while $\pi_{0}$ is a product of $\lfloor n / 2\rfloor$ disjoint transpositions, and hence $\pi_{0}^{I}=\pi_{0}$. Hence the orbit of $\pi_{0}$ under $G$ is the set $\left\{\iota, \pi_{0}\right\}$ and this is also the orbit of $\iota$. The orbit of $\pi_{1}$ under $G$ is disjoint from that of $\pi_{0}$ and $\iota$. It consists of $\pi_{1}, \pi_{1}^{-1}, \pi_{1}^{R}=\pi_{1}^{C}=1 n \ldots 2, \pi_{1}^{-R}=(n-1) \ldots 1 n$.

It follows that if $S \circ g=$ DIS for some permutation statistic $S$ and element $g \in G$, then $S\left(\pi_{0}\right)$ must equal zero or DIS $\left(\pi_{0}\right)$. However it is readily seen by comparing with Table 2 that none of the statistics in [CSZ97] satisfy this property. This includes those mentioned in passing, such as LAG and SIST.

Now consider the statistics in [BS00, Table 1], given in terms of permutation pattern counts. Any pattern that is not strictly descending does not occur in $\pi_{0}$, so we need only count occurrences of $b a, c b a, c b-a, c-b a$. Again, none of these lead to zero or $\operatorname{DIS}\left(\pi_{0}\right)$, since the number of occurrences of these four patterns in $\pi_{0}$ is respectively $n-1, n-2,(n-1)(n-2) / 2,(n-1)(n-2) / 2$.

Finally we consider Haglund's statistic HAG and a descent-based variant DAG as defined in [BS00]. The statistic DAG can be dealt with by counting pattern occurrences in $\pi_{0}$ as above. However it is not as easy to differentiate HAG from DIS by using our special permutations. In fact when $n$ is even, HAG and DIS take the same value on $\pi_{0}$ (they coincide with Edif). When $n$ is odd, HAG is smaller than DIS by $(n-1) / 2$. We instead use the permutation $\pi_{2}=n 2 \ldots(n-1) 1$ formed from $\iota$ by a single transposition. Its orbit under $G$ consists of itself and its reverse $1(n-1) \ldots 2 n$, and DIS takes the values $n-1$ and $\left\lceil(n-2)^{2} / 4\right\rceil$ respectively on these two elements. However, $\operatorname{HAG}\left(\pi_{2}\right)=1$.

\subsection{Euler-Mahonian pairs and nontrivial bijections}

In [CSZ97] a bijection $\Phi$ of $\mathrm{S}_{n}$ was given and it was shown that $\Phi$ had appeared (somewhat disguised) in several previous papers. The key property of $\Phi$ is that it takes (des, Dbot, Ddif, Res) to (exc, Ebot, Edif, Ine). This then gives access to equidistribution results for Euler-Mahonian pairs. The term Euler-Mahonian refers in the literature to a bistatistic $(e, M)$ such that $e$ is Eulerian, $M$ is Mahonian, and the joint distribution of $(e, M)$ is the same as that of another well-known pair $\left(e^{\prime}, M^{\prime}\right)$. Originally the term was used only for $\left(e^{\prime}, M^{\prime}\right)=($ des, MAJ). Other authors, for example [BS00, CSZ97] allow more possibilities for $\left(e^{\prime}, M^{\prime}\right)$, and aim to classify these bistatistics up to equidistribution.

In [BS00, Table 2] seven equivalence classes (under equidistribution) of EulerMahonian pairs (des, $T$ ) were given for $n=5$ (note that the second matrix, corresponding to MAJ, has an error: in the row indexed by des $=2$, the entries listed as 14 should be 16). This corresponds to 14 Mahonian statistics $T$. It is easy to see that DIS does not occur in this table, because its maximum value occurs on $\pi_{1}$ and $\operatorname{des}\left(\pi_{1}\right)=1$, yet none of the seven distributions has a nonzero entry in the $(1,10)$ position. We can also check easily that (exc, DIS) has a different distribution from all the entries in the table. Thus if $T^{\prime}$ is the image of such a $T$ under $\Phi$, then $T^{\prime} \neq$ DIS. 


$\begin{array}{ccccccccccc}1 & 0 & 0 & 0 & 0 & 0 & 0 & 0 & 0 & 0 & 0 \\ 0 & 4 & 6 & 8 & 8 & 0 & 0 & 0 & 0 & 0 & 0 \\ 0 & 0 & 3 & 7 & 10 & 22 & 15 & 9 & 0 & 0 & 0 \\ 0 & 0 & 0 & 0 & 2 & 0 & 5 & 6 & 9 & 4 & 0 \\ 0 & 0 & 0 & 0 & 0 & 0 & 0 & 0 & 0 & 0 & 1 \\ & & & & & & & & & & \\ 1 & 0 & 0 & 0 & 0 & 0 & 0 & 0 & 0 & 0 & 0 \\ 0 & 4 & 3 & 5 & 3 & 3 & 3 & 2 & 1 & 1 & 1 \\ 0 & 0 & 6 & 6 & 13 & 12 & 9 & 9 & 8 & 3 & 0 \\ 0 & 0 & 0 & 4 & 3 & 7 & 8 & 4 & 0 & 0 & 0 \\ 0 & 0 & 0 & 0 & 1 & 0 & 0 & 0 & 0 & 0 & 0\end{array}$

Table 3: Joint distributions (exc, DIS) and (exc, IDIS) for $n=5$.

$\begin{array}{ccccccccccc}1 & 0 & 0 & 0 & 0 & 0 & 0 & 0 & 0 & 0 & 0 \\ 0 & 4 & 3 & 5 & 5 & 2 & 3 & 2 & 1 & 0 & 1 \\ 0 & 0 & 6 & 8 & 12 & 14 & 11 & 7 & 5 & 3 & 0 \\ 0 & 0 & 0 & 2 & 3 & 6 & 5 & 6 & 3 & 1 & 0 \\ 0 & 0 & 0 & 0 & 0 & 0 & 1 & 0 & 0 & 0 & 0 \\ & & & & & & & & & & \\ 1 & 0 & 0 & 0 & 0 & 0 & 0 & 0 & 0 & 0 & 0 \\ 0 & 4 & 3 & 5 & 5 & 2 & 2 & 3 & 1 & 0 & 1 \\ 0 & 0 & 6 & 8 & 12 & 15 & 11 & 6 & 5 & 3 & 0 \\ 0 & 0 & 0 & 2 & 3 & 5 & 6 & 6 & 3 & 1 & 0 \\ 0 & 0 & 0 & 0 & 0 & 0 & 1 & 0 & 0 & 0 & 0\end{array}$

Table 4: Joint distributions (des, DIS) and (des, IDIS) for $n=5$.

We still need to check HAG. By direct computation we can show readily that

$$
\begin{aligned}
& \Phi\left(\pi_{0}\right)=\pi_{1} \\
& \Phi\left(\pi_{1}\right)=\left(\pi_{1}\right)^{-1}
\end{aligned}
$$

Suppose that $T=\mathrm{DIS} \circ \Phi$ for some $T \in \Sigma$. Then $T\left(\pi_{0}\right)=n(n-1) / 2$ and $T\left(\pi_{1}\right)=n-1$. Clearly HAG fails this test. If $T \circ \Phi=$ DIS then $T\left(\pi_{1}\right)=\left\lceil n^{2} / 4\right\rceil$ which again HAG fails.

\section{An Eulerian partner for DIS}

Skandera [Ska02] gave a general procedure for associating to each Mahonian statistic $M$ another statistic $e$ that is Eulerian and such that the pair $(e, M)$ is Euler-Mahonian 
(equidistributed with (des, MAJ)). Of course, such an Eulerian statistic may not be known or particularly interesting.

Applying this procedure to DIS yields an Eulerian statistic eul. Concretely, eul $(\pi)$ is obtained from the numbers $d_{i}$ by listing them in order, and counting each time we encounter a number larger than the current record (the record being initialized to zero). For example, for our running example 25413 we have $d=(0,1,2,3,2)$ and so eul takes the value 3. Also note that $\operatorname{eul}\left(\pi_{0}\right)=\lceil(n-1) / 2\rceil$ while $\operatorname{eul}(\iota)=0$ and $\operatorname{eul}\left(\pi_{1}\right)=n-1$.

A well-known Eulerian statistic is the number of excedances exc. Now exc agrees with eul on $\pi_{0}$ and $\pi_{1}$. Also, eul and exc are equal when $n=3$. Nevertheless, eul is not equal to exc in general, nor does it equal des.

Eulerian statistics in the literature are less commonly found than Mahonian ones. As far as I am aware, eul is itself new, but this is based on much less evidence than the corresponding claim about DIS.

\section{Further comments}

The current paper gives substantial evidence that the statistic DIS is really new. In order to check thoroughly whether a permutation statistic is new to the literature, one would ideally check a database of such statistics. I have not found such a database. I propose that as a minimum, tables of values for $n=4$, along with the joint distribution with exc and des for $n=5$, be included in all papers dealing with this topic, to allow easy comparison. It would then be much easier to show that the entire group $\Gamma$ generated by $G$ and $\Phi$ does not have any element $g$ with $T \circ g=$ DIS for some known Mahonian $T$, since all such $T$ of which I am aware are consistent.

It may be desirable to find a "static" description of DIS and IDIS, which have been defined "dynamically". I do not know a systematic way to do this (one possible idea is to find linear combinations of the above partial statistics that fit the values for small $n$ ). A related question is to determine whether DIS can be written as a Mahonian $d$-function for some $d$.

The statistic IDIS should extend to words via the selection sort interpretation. Whether this statistic is Mahonian on words should be investigated and I intend to do this in future work. Dennis White has informed me that a general machine explained by him and Jennifer Galovich in [GW96] will construct a bijection between (nearly) any Mahonian statistic and INV. The machine works in the context of words, and this connection may be worth pursuing.

Note: As I was preparing this article I was made aware of completely independent recent work by T. Kyle Petersen [Pet10] that also discusses the statistic DIS and some generalizations. The intersection between the topics of these two papers is small, and the reader should consult both articles for a fuller picture. 


\section{References}

[BS00] Eric Babson and Einar Steingrímsson, Generalized permutation patterns and a classification of the Mahonian statistics, Sém. Lothar. Combin. 44 (2000), Art. B44b, 18 pp. (electronic).

[CSZ97] R.J. Clarke, E. Steingrmsson, and J. Zeng, New Euler-Mahonian statistics on permutations and words, Advances in Applied Mathematics 18 (1997), no. 3, $237-270$.

[Dur64] R. Durstenfeld, Algorithm 235: Random permutation, Comm. Assoc. Comput. Mach. 7 (1964), 420.

[FY38] R. A. Fisher and F. Yates, Statistical tables, Oliver and Boyd, 1938.

[GW96] J. Galovich and D. White, Recursive statistics on words, Discrete Mathematics 157 (1996), no. 1-3, 169-191.

[Knu81] Donald E. Knuth, The art of computer programming. Vol. 2, second ed., Addison-Wesley Publishing Co., Reading, Mass., 1981.

[Pet10] T. K. Petersen, The sorting index, ArXiv e-prints 1007.1207.

[Ska02] Mark Skandera, An Eulerian partner for inversions, Sém. Lothar. Combin. 46 (2001/02), Art. B46d, 19 pp. (electronic).

[Wil09] M.C. Wilson, Random and exhaustive generation of permutations and cycles, Annals of Combinatorics 12 (2009), no. 4, 509-520. 